\title{
Search for New Phenomena at the High Luminosity LHC with ATLAS
}

$17^{\text {th }}$ Lomonosov Conference on Elementary Particle Physics $20^{\text {th }}$ August 2015

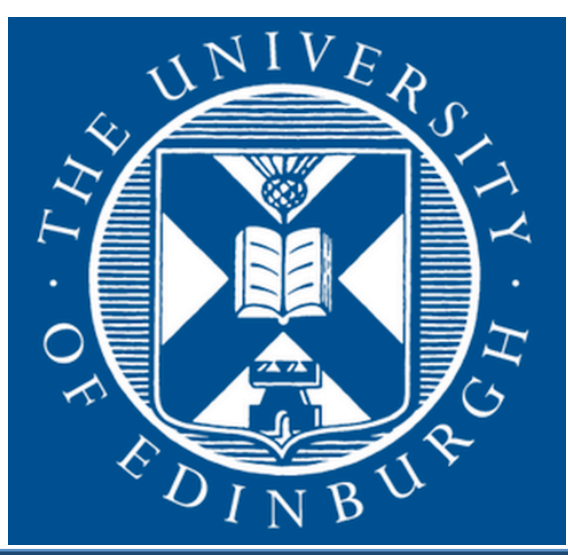

\author{
Paul Glaysher \\ (University of Edinburgh) \\ On behalf of the ATLAS collaboration
}

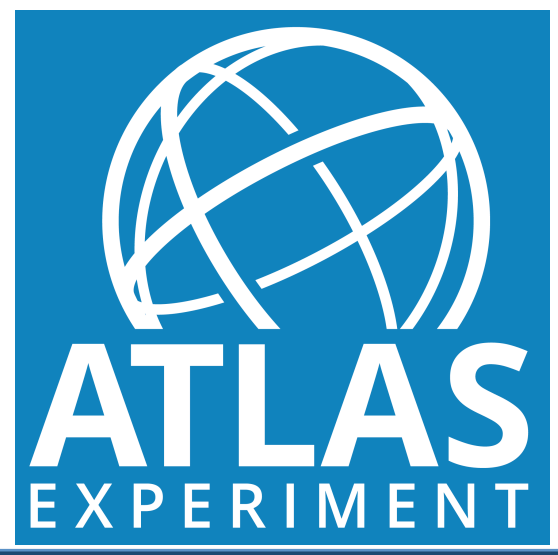

$17^{\text {th }}$ Lomonosov Conference, Moscow - Paul Glaysher 


\section{Introduction}

- The LHC is the primary discovery tool of our time

- The Higgs boson discovered at ATLAS and CMS was the highlight of the first LHC run

$>$ All evidence points to a $125 \mathrm{GeV}$ Higgs boson, compatible with the Standard Model (SM)

- What will we see next, BSM physics?

- An upgrade to the LHC (High Luminosity-LHC) is required to fully exploit its discovery potential

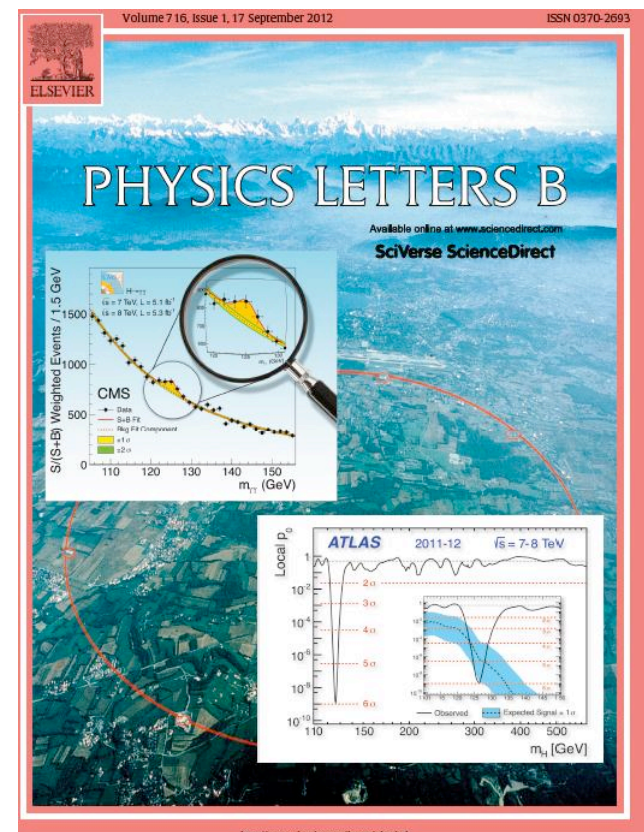

- This talk covers prospects and sensitivity studies by ATLAS for discovery potential at the HL-LHC 


\section{High Luminosity LHC}

https://cds.cern.ch/record/1975962

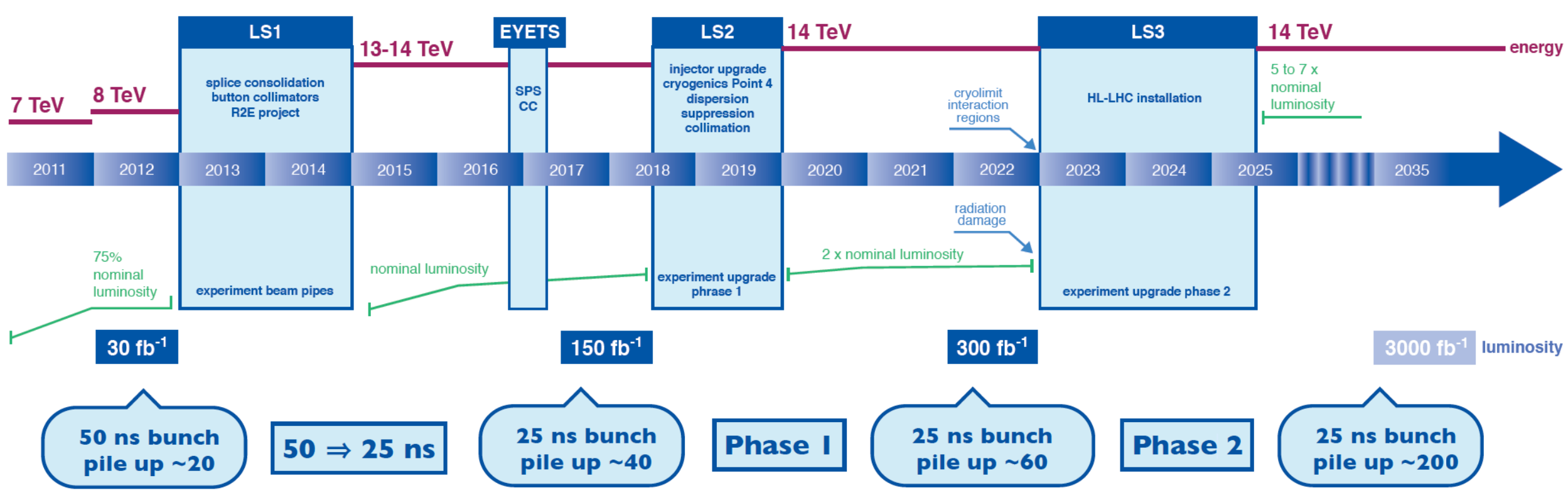

- Pile up: number of simultaneous interactions per bunch crossing

- Increases with instantaneous luminosity

- Degrades experimental environment

$>$ Primary challenge for a detector at HL-HLC 


\section{ATLAS Detector Upgrade}

Phase 1 upgrades:

- Fast tracker algorithms (FTK)

- Extend muon spectrometer endcaps, New Small Wheel (NSW)

- Improve calorimeter readout
Upgrades to the ATLAS detector are essential to pursue rich physics program at the HL-LHC

- Mitigate pile up effects

- Address radiation damage to sensors
Phase 2 upgrades:

- Redesign of trigger system

- All silicon inner tracker (ITK)

- Calorimeter electronics and forward region (sFCal or Mini-Fcal)

- Muon system

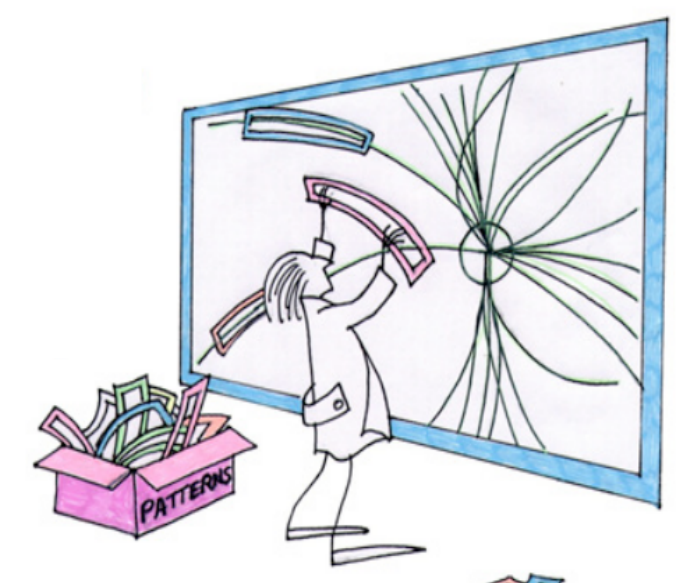

Phase 0+1 FTK: cds.cern.ch/record/1552953 Image: cds.cern.ch/record/2002880/

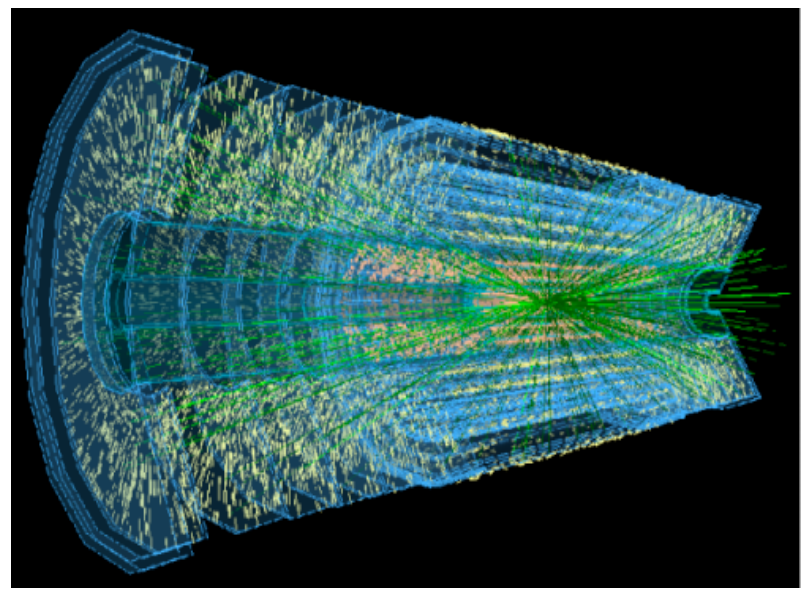

Phase 2 tracker simulation: atlas.desy.de/activities/tracker_upgrade/ 


\section{Simulating future ATLAS Detector}

ATLAS HL studies are performed using particle level Monte Carlo

- Detector response functions based on full Geant4 simulations are applied

- Based on an upgraded ATLAS detector and takes impact of future pile up scenarios into account

$>$ Assumes current or even improved detector performance despite high pile up

- Scenarios with improved systematic uncertainties over today's understanding are considered

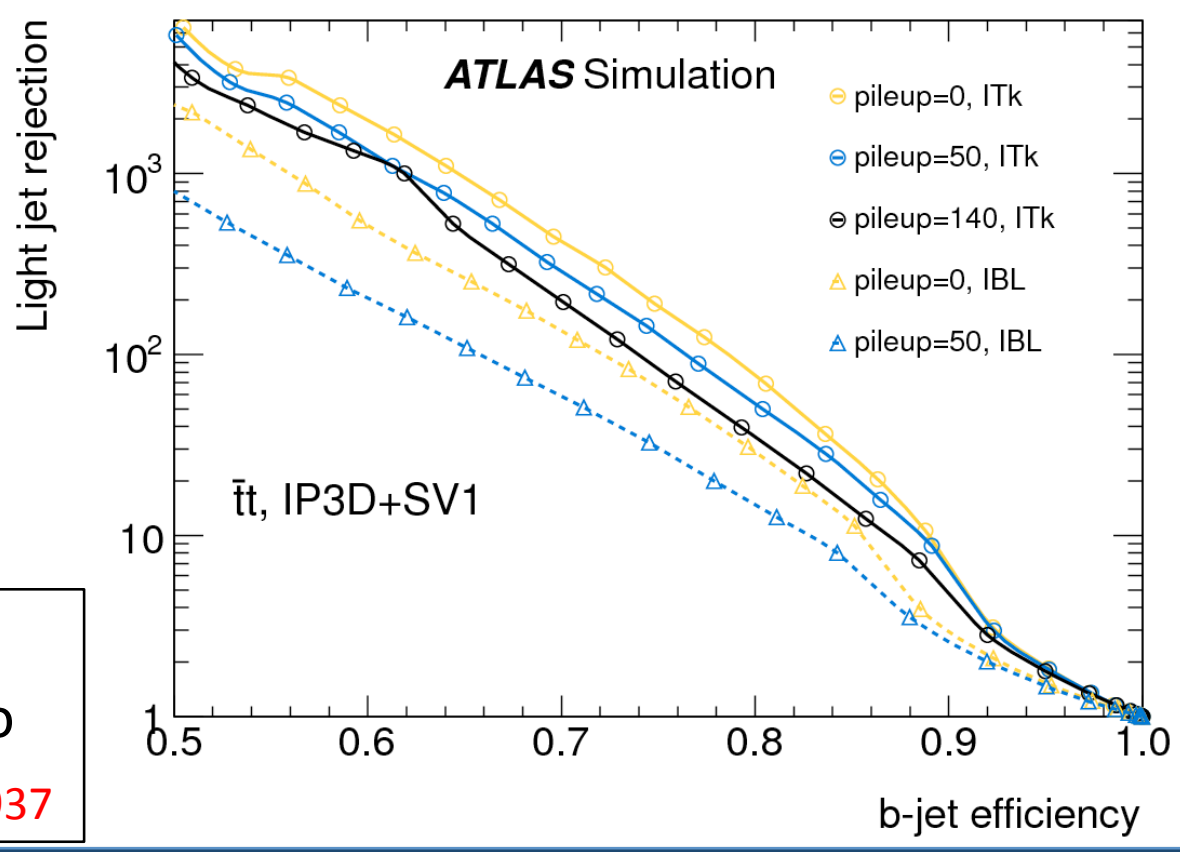

Expected improvements in b-tagging performance with the Itk upgrade compared to the current detector 


\section{New Phenomena}

- ATLAS has conducted many studies into the prospects and discovery potential of many BSM and New Phenomena cases at the HL-LHC

- A selection is presented here:

- Higgs sector

- Supersymmetry

- Gluino, squark and $\chi \pm \chi^{0}$, searches

- Di-jet resonance searches

- Flavour Changing Neutral Currents 


\section{HL-LHC: A Higgs Boson Factory}

\section{Higgs Physics goals}

- Rare decays and coupling

- Higgs pair production

- Higgs boson as a portal to new physics

- More Higgs-like particles

- Two Higgs doublet Model, MSSM

- Higgs compositeness

- Coupling to Dark Matter

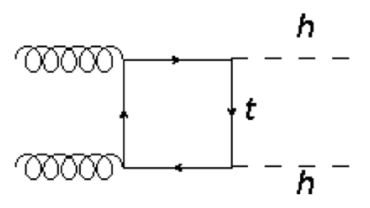

I $25 \mathrm{GeV}$ Higgs bosons at $\sqrt{ } \mathrm{s}=14 \mathrm{TeV} 3000 \mathrm{fb}^{-1}$

\begin{tabular}{c|c} 
HL-LHC total & $170 \mathrm{M}$ \\
VBF & $13 \mathrm{M}$ \\
$\mathrm{ttH}$ & $1.8 \mathrm{M}$ \\
$\mathrm{H} \rightarrow \mathrm{ZY}$ & $230 \mathrm{k}$ \\
$\mathrm{H} \rightarrow \mu \mu$ & $37 \mathrm{k}$ \\
$\mathrm{HH}$ & $121 \mathrm{k}$
\end{tabular}

Number of event of different Higgs processes expected in the HL-LHC dataset
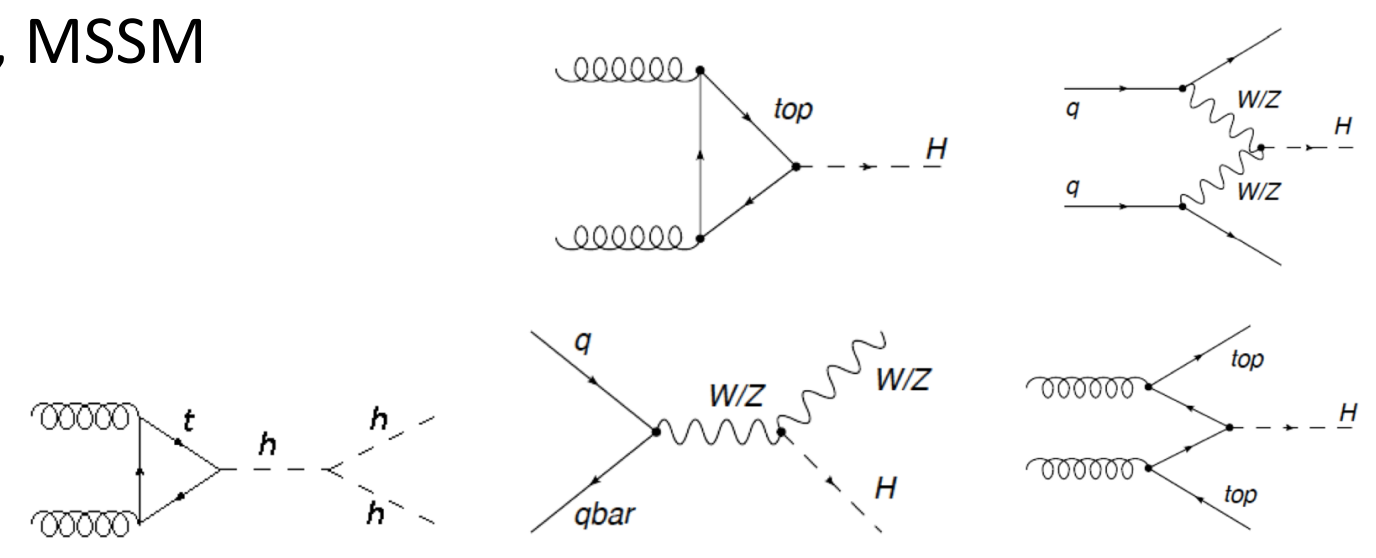
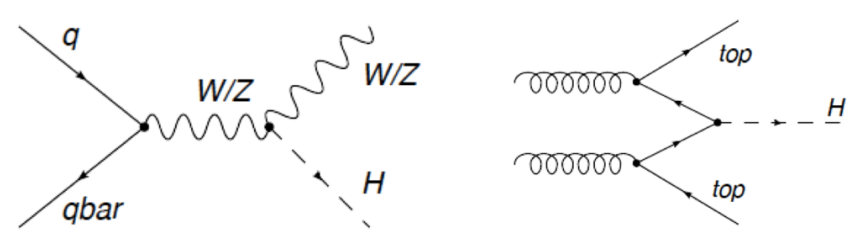


\section{Higgs to SM particle couplings}

Measure couplings strength in units of SM, leading order expectation

$$
\mu=\frac{(\sigma \times B R)_{\text {obs }}}{(\sigma \times B R)_{S M}}
$$

ATLAS Simulation Preliminary

is $=14 \mathrm{TeV}: \int \mathrm{Ldt}=300 \mathrm{fb}^{-1} ; \int \mathrm{Ldt}=3000 \mathrm{fb}^{-1}$

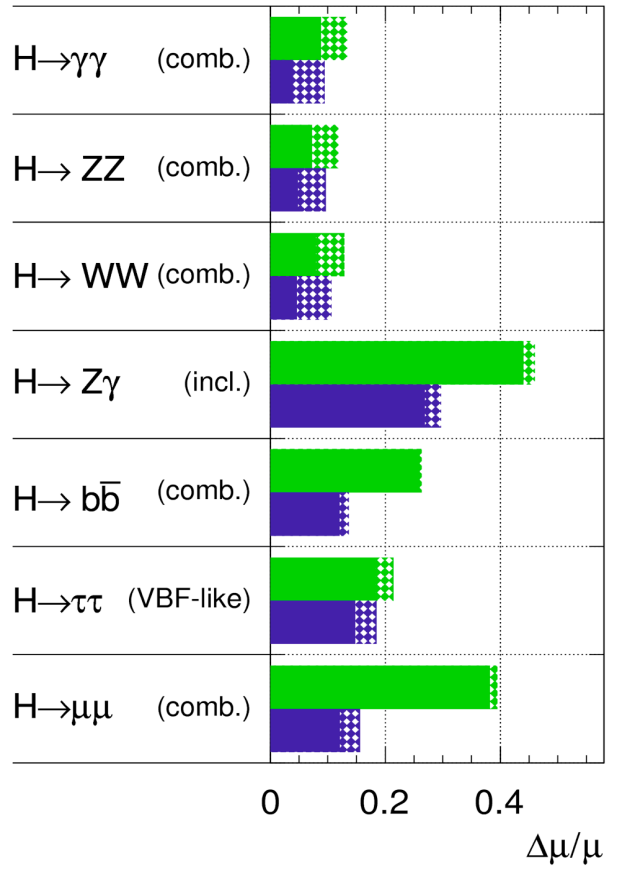

- $\mathrm{m}_{\mathrm{H}}=125 \mathrm{GeV}$, narrow width approximation

- Measure couplings within 2-15\% precision

$>$ Will be able to quantify small deviations from the SM

Outstanding SM measurements of Higgs couplings to:

- $\mathrm{H}->$ bb large background

- Expect to observe in Run 2

- $\quad \mathrm{H}->\mu \mu$ low branching ratio

$>$ Expect to observe at HL-LHC
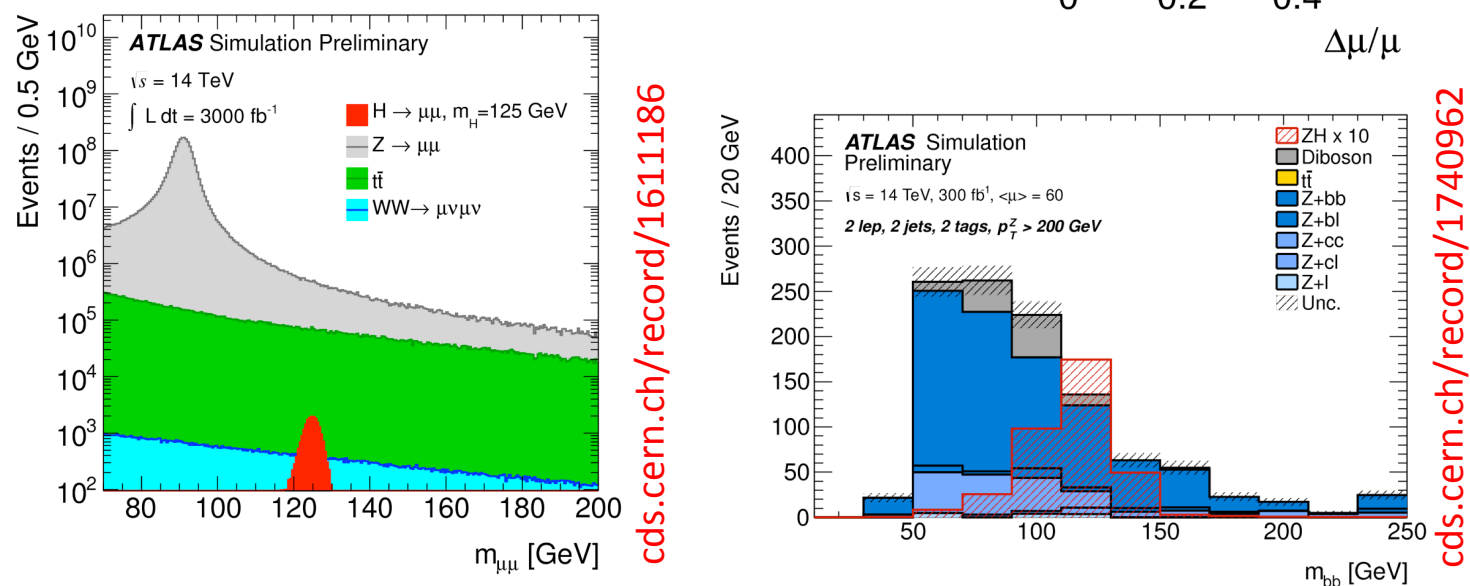


\section{Di-Higgs Production}

- Sensitive to the Higgs self-coupling $\boldsymbol{\lambda}$, this challenging measurement will provide strongest test of SM nature of the Higgs boson

- $\mathrm{SM}$ cross section at Vs $=14 \mathrm{TeV} 40.7 \mathrm{fb}$ (NNLO) suffers from destructive interference
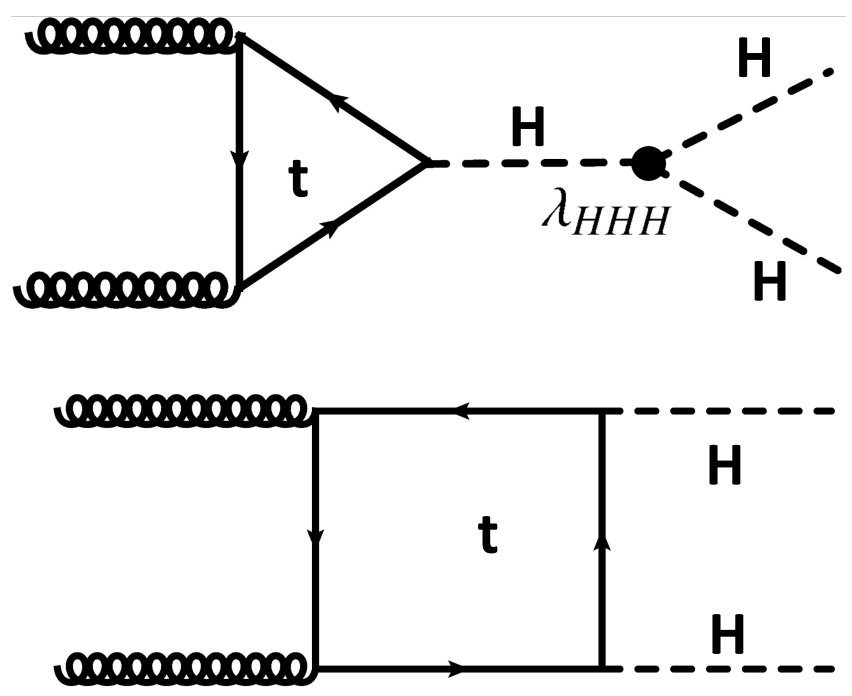

Final states studied:

- HH -> bbpץ : 320 events expected - clean signal

- HH ->WWyy : 30k events expected - large backgrounds

- bbbb and bbrt final states are also under consideration 


\section{$\mathrm{HH} \rightarrow$ bbyy}

- $\quad$ Expect 8.4 signal and $\sim 47$ bkg events with a $S / V B \sim 1.3$

- BSM models with $\lambda / \lambda_{S M}<-1.3 \& \lambda / \lambda_{S M}>8.7$ can be excluded with $3000 \mathrm{fb}^{-1}$

$>$ Combination with other channels (ATLAS +CMS) likely to be sensitive to SM Higgs self-coupling

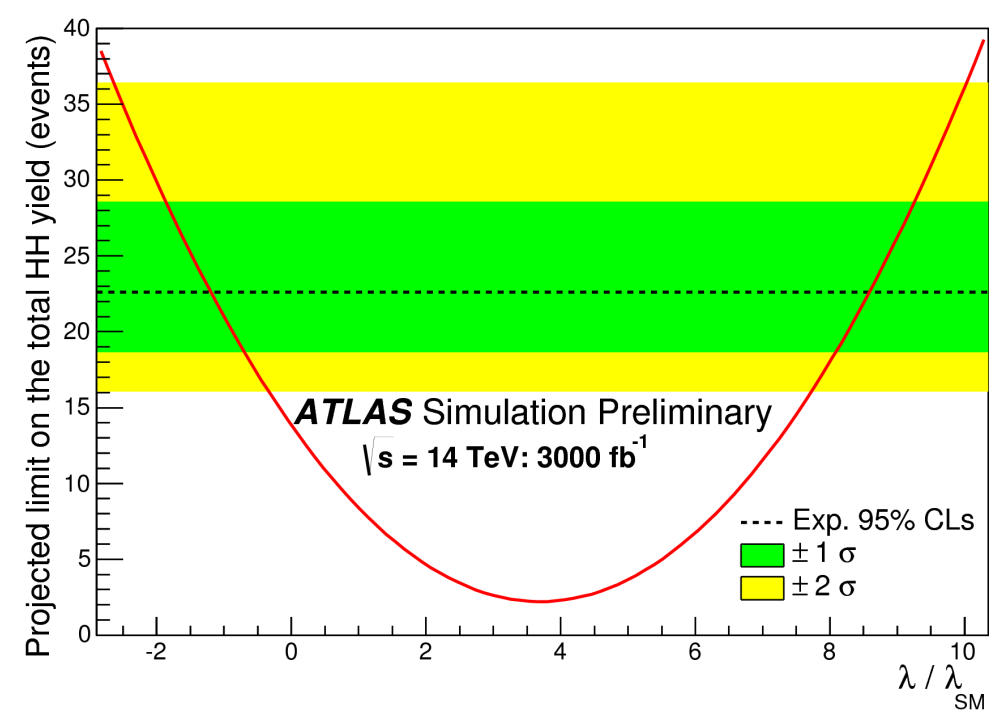

Number of signal events (red) as a function of $\lambda / \lambda_{S M}$, with projected sensitivity of the $3000 \mathrm{fb}^{-1}$ dataset
- Angular cuts bring strong separation of signal and background

- 'Others' includes cc $\gamma \gamma$, bbyj, bbjj and jjpy, where $\mathrm{j}$ signifies a jet.

cds.cern.ch/record/1956733
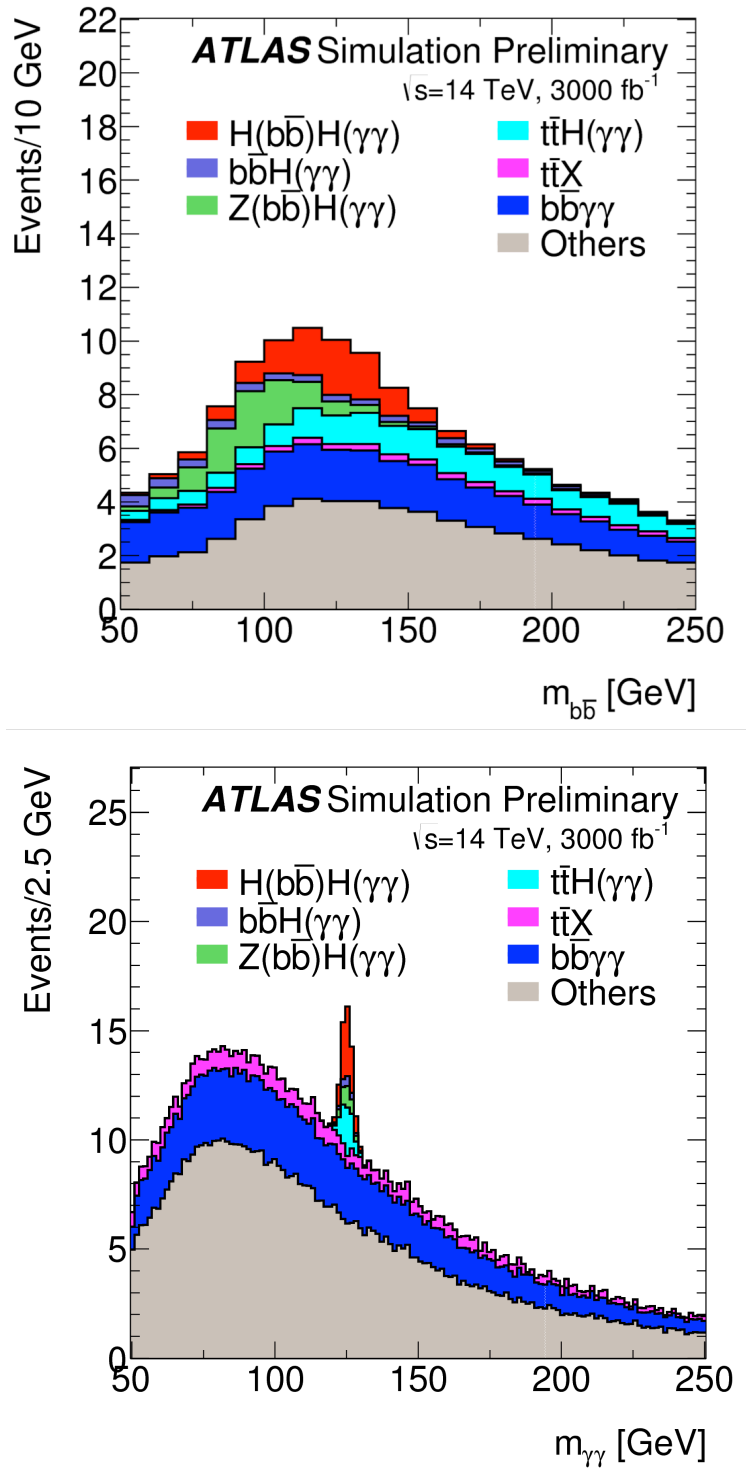


\section{Two Higgs Doublet Models (2HDM)}

- Higgs sector extended by an additional doublet, resulting in a model with five Higgs bosons:

$>$ one neutral-CP-odd $\mathbf{A}$ and two charged $\mathbf{H}^{ \pm}$

$>$ Two neutral-CP-even: $h_{m=125 ~ G e v}$ and a heavy $\mathbf{H}$

Indirect search for $\mathrm{H}$ through precision measurements of $h$ couplings:

ATLAS sensitive to $H$ via mixing with $h$

a mixing angle of $\mathrm{H}$ and $\mathrm{h}$

$\tan \beta=$ ratio of the vacuum expectation values of the two doublets: relative coupling strength of $\mathbf{H} / \mathbf{h}$

$\operatorname{Cos}(\beta-\alpha)$ couplings and mixing term. $A$ measure of relative coupling strength of H->VV over SM expectation

\section{Exp}

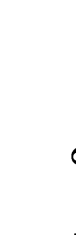

2HDM Type II $\boldsymbol{A} \boldsymbol{T L A S}$ Simulation

Exp. $95 \% \mathrm{CL}$ at $\mathrm{s}=14 \mathrm{TeV} \quad$ Preliminary

- $-\mathrm{SM}$

L $300 \mathrm{fb}^{-1}$. All unc.

- - - - Ldt $=300 \mathrm{fb}^{-1}:$ No theo.

Ldt $=3000 \mathrm{fb}^{-1}$ : All unc.

Combined

$\mathrm{h} \rightarrow \gamma \gamma, \mathrm{ZZ}^{*}, \mathrm{WW}^{*}$

$\mathrm{h} \rightarrow \mathrm{Z} \gamma, \mu \mu, \tau \tau, \mathrm{bb}$

- - - - J Ldt $=3000 \mathrm{fb}^{-1}$ : No theo.

$\stackrel{\infty}{\frac{\infty}{\varpi}}$

${ }_{3}$

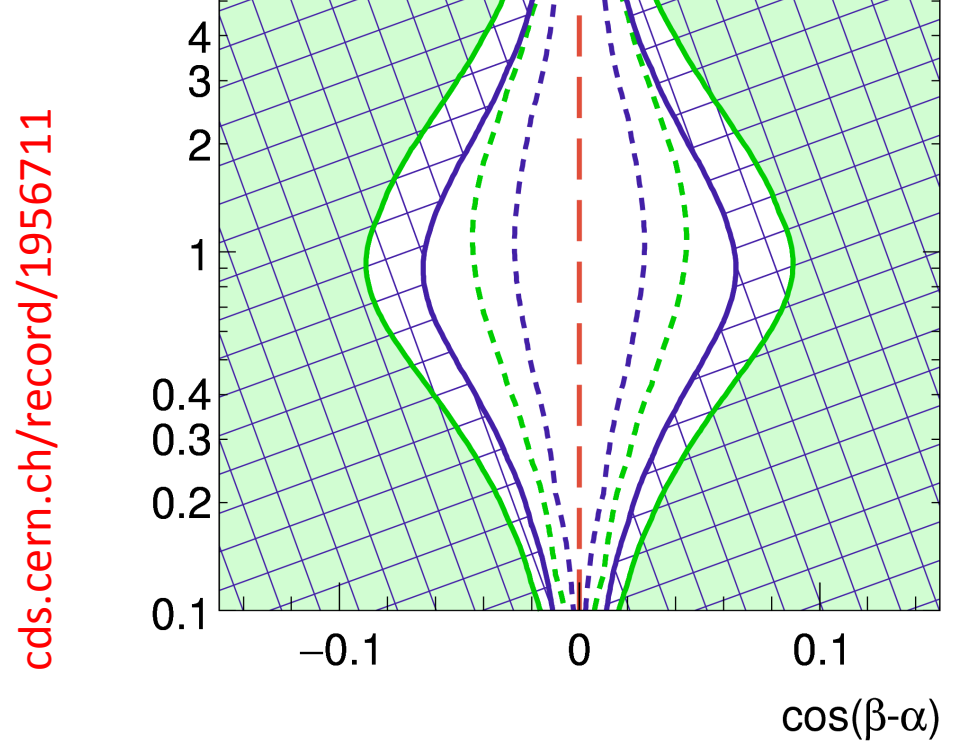

Excluded regions of $\operatorname{Cos}(\beta-\alpha)$ vs $\tan \beta$ of the type II $2 \mathrm{HDMs}$ by fits to the measured rates of Higgs boson production and decays. 


\section{Simplified MSSM}

- A concrete example of 2HDM: Minimal Super-symmetric Model

- Simplified MSSM:

$-\mathbf{H}$ and $\mathbf{h}$ mixing is fully determined by $\mathrm{m}_{\mathbf{A}}$ and $\tan \boldsymbol{\beta}$

cds.cern.ch/record/1956711

- SM Higgs boson coupling rates $\mathrm{K}_{\mathrm{V}}, \mathrm{K}_{\mathrm{u}}, \mathrm{K}_{\mathrm{d}}$ would be modified by $\mathrm{m}_{\mathrm{A}}$

- Simplified model serves as good benchmark study

- Particularly useful if no direct SUSY observation is made

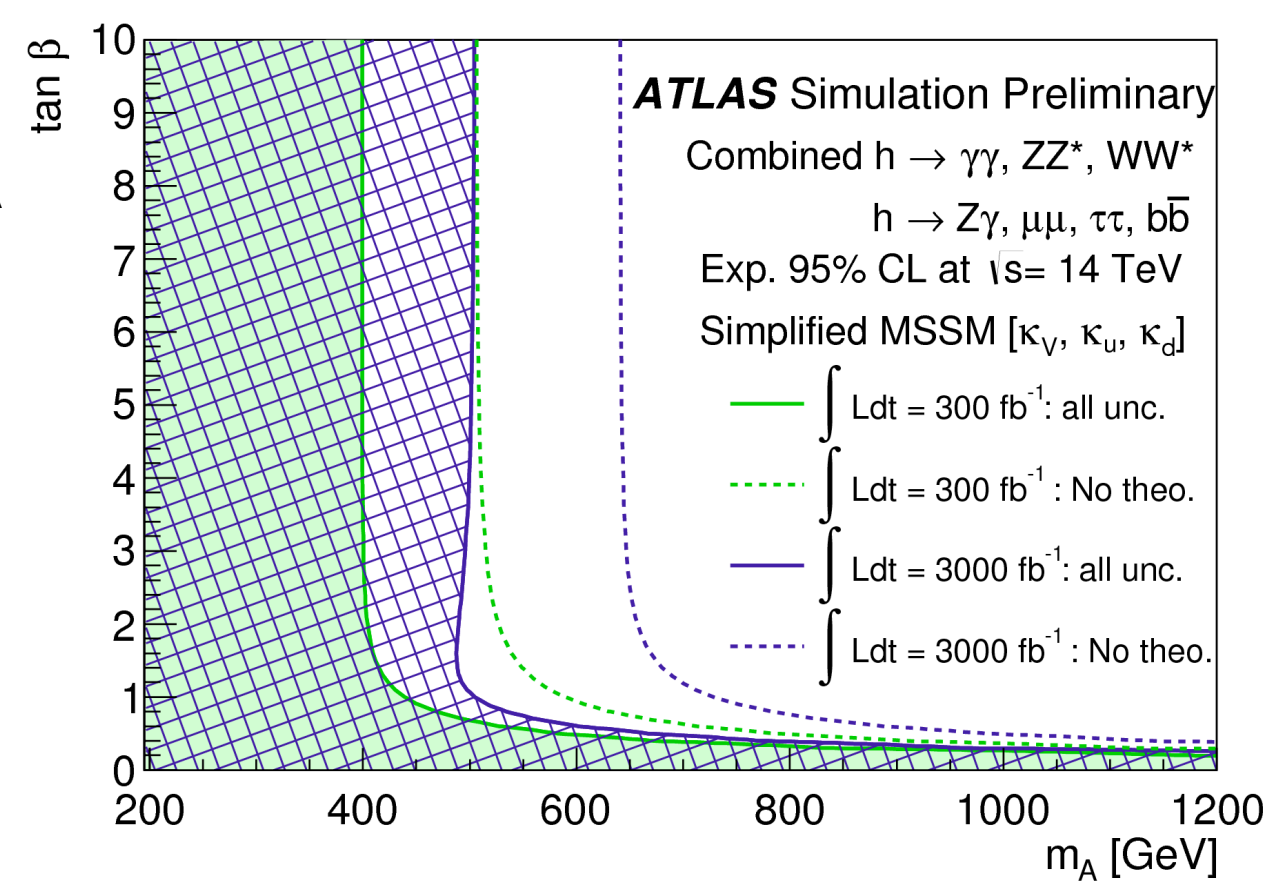

Regions $m_{A} v s \tan \beta$ expected to be excluded in a simplified MSSM model via fits to the measured rates of Higgs boson production and decays. 


\section{Minimal Composite Higgs Model}

\section{For a pseudo-Nambu-Goldstone, composite Higgs boson:}

- Higgs coupling modified as a function of the compositeness $f$

$$
>\xi=v^{2} / f^{2}
$$

- MCHM4:

$$
>K=K_{V}=K_{F}=V(1-\xi)
$$

- MCHM5:

$$
>\kappa_{v}=V(1-\xi) ; \kappa_{F}=1-2 \xi / V(1-\xi)
$$

Expected 95\% CL lower limit on Higgs boson compositeness scale f:

\begin{tabular}{ccccc}
\hline \hline Model & \multicolumn{2}{c}{$300 \mathrm{fb}^{-1}$} & \multicolumn{2}{c}{$3000 \mathrm{fb}^{-1}$} \\
& All unc. & No theory unc. & All unc. & No theory unc. \\
\hline MCHM4 & $620 \mathrm{GeV}$ & $810 \mathrm{GeV}$ & $710 \mathrm{GeV}$ & $980 \mathrm{GeV}$ \\
MCHM5 & $780 \mathrm{GeV}$ & $950 \mathrm{GeV}$ & $1.0 \mathrm{TeV}$ & $1.2 \mathrm{TeV}$ \\
\hline \hline
\end{tabular}

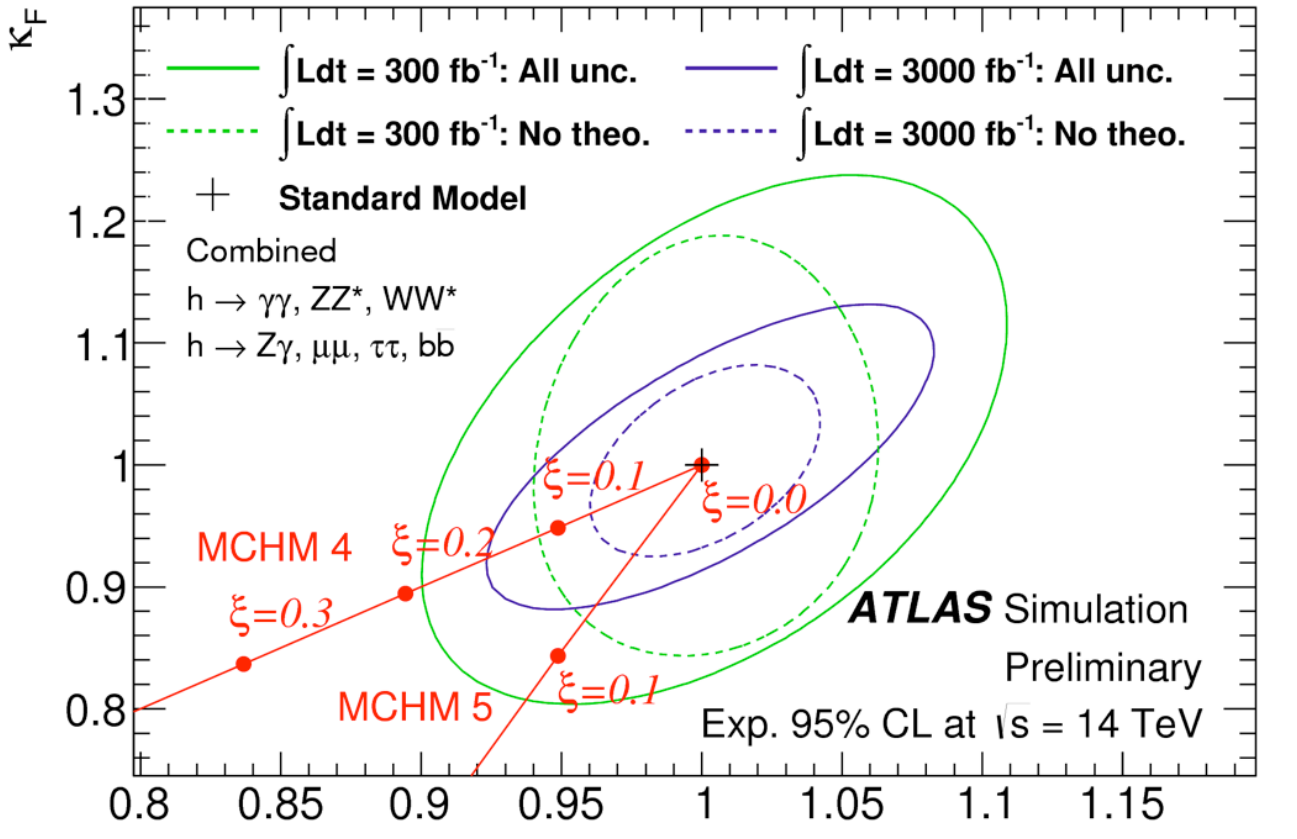

$\kappa_{\mathrm{v}}$ 


\section{Higgs Portal to Dark Matter}

- Assuming a Wimp as a DM candidate, which interacts very weakly with SM particles except for the Higgs boson

- Expected 95\% CL upper limit BR $\left(\mathrm{H} \rightarrow\right.$ invisible) at $3000 \mathrm{fb}^{-1}$

- $\mathrm{BR}_{\text {inv }}<0.13(0.09)$, nominal (improved) systematics scenario

- Run 1 VBF Higgs channel set upper limit of $\mathrm{BR}_{\mathrm{inv}}<0.29$

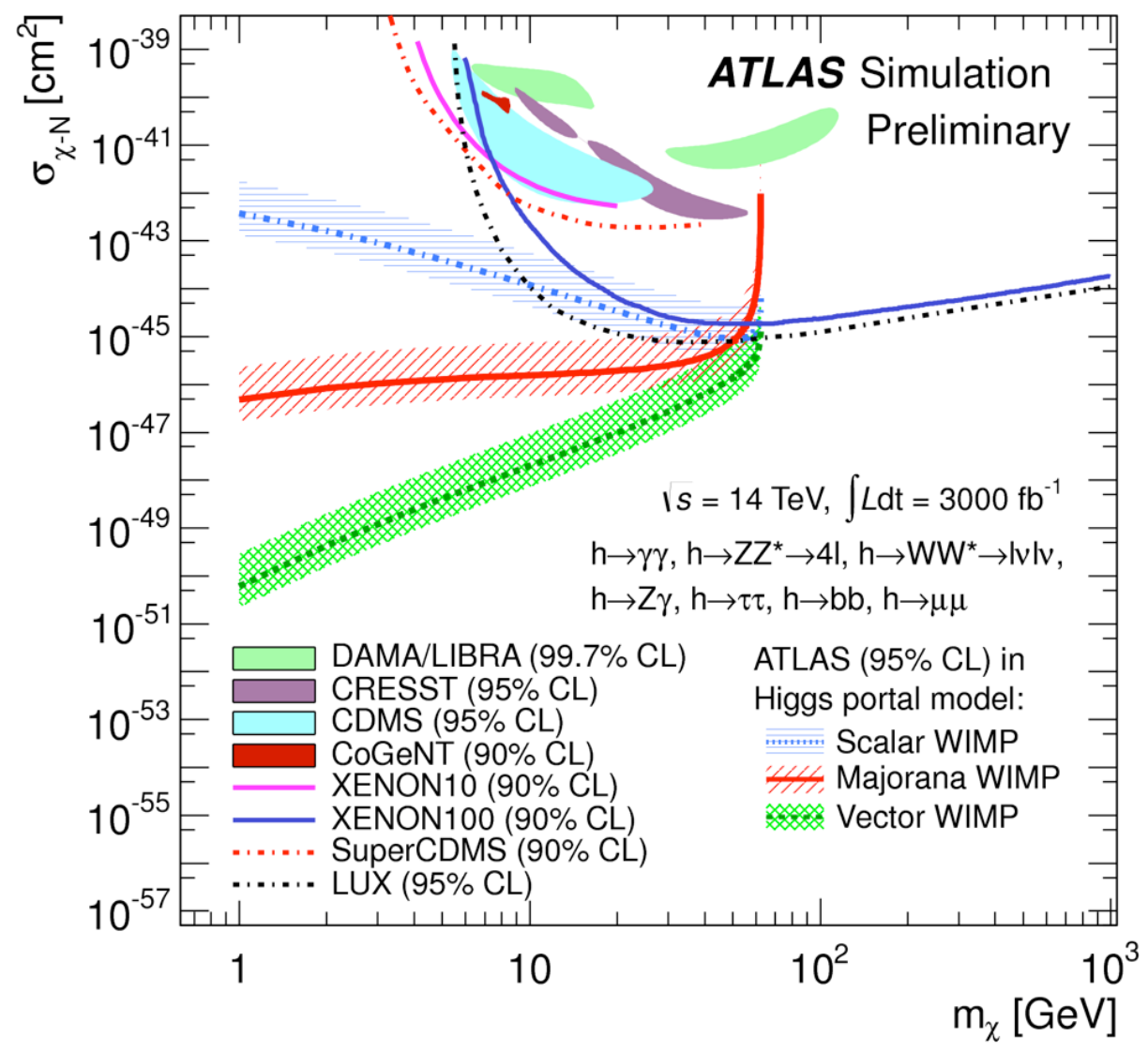

cds.cern.ch/record/1956711/

$>$ ATLAS will provide complimentary measurement to those from dedicated DM experiments 


\section{SUSY at the HL-LHC}

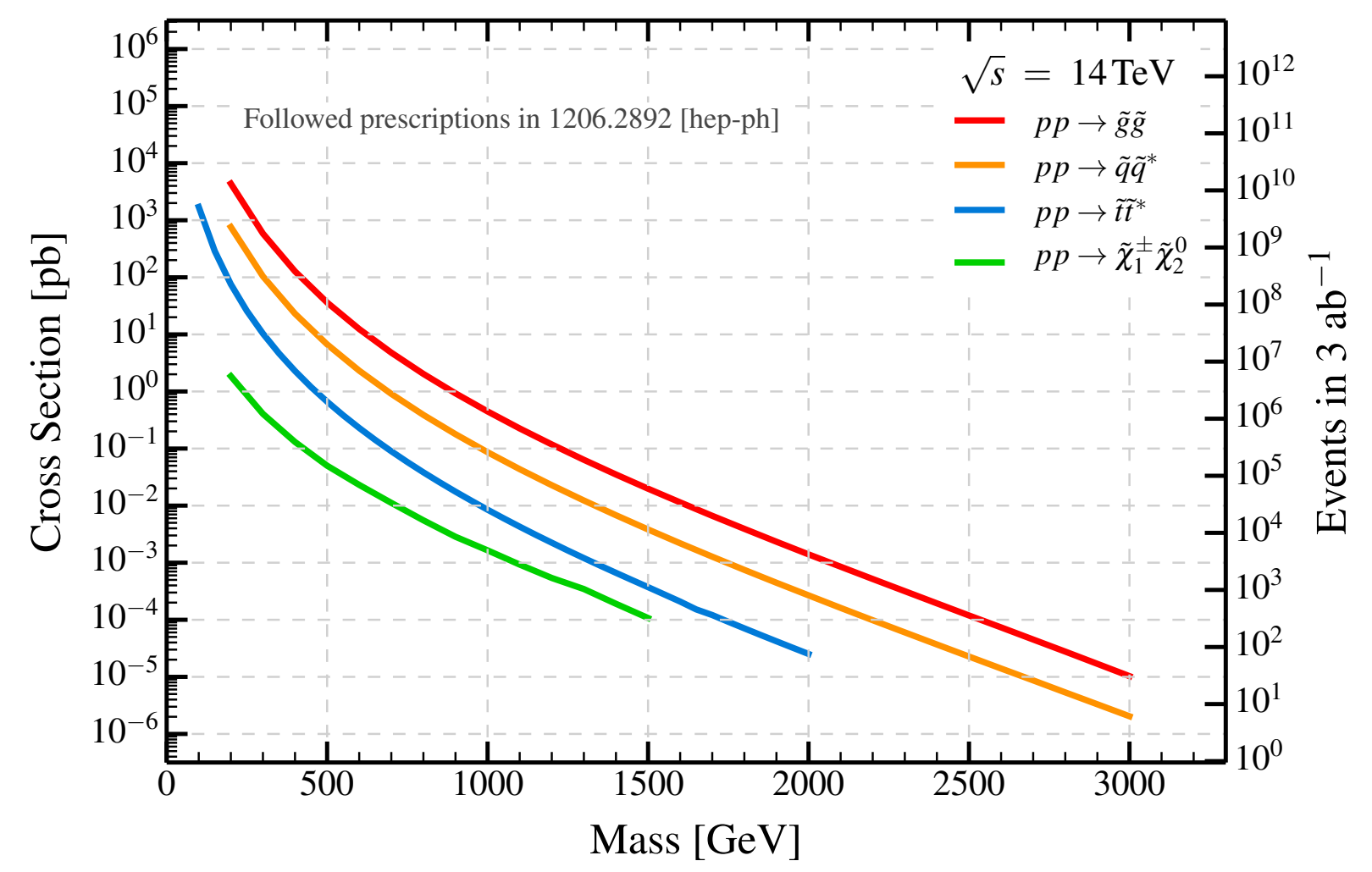

NLO cross-sections as a function of the average mass of the pair-produced SUSY particles.

The chargino and neutralino-2 are assumed to be wino-like.

Studies assume R-parity conserving SUSY and a stable LSP.

- Supersymmetry is one of the best motivated and wellstudied extensions of the Standard Model

- The HL-HLC will allow to probe small cross section processes 


\section{gluino pair production}

- Assumes $\operatorname{BR}\left(\tilde{g} \rightarrow q \chi_{1}^{0}\right)=100 \%$

- Signature:

- No leptons, 4 jets and $\mathrm{E}_{\mathrm{T}}^{\text {missing }}>160 \mathrm{GeV}$

- Dominant backgrounds:

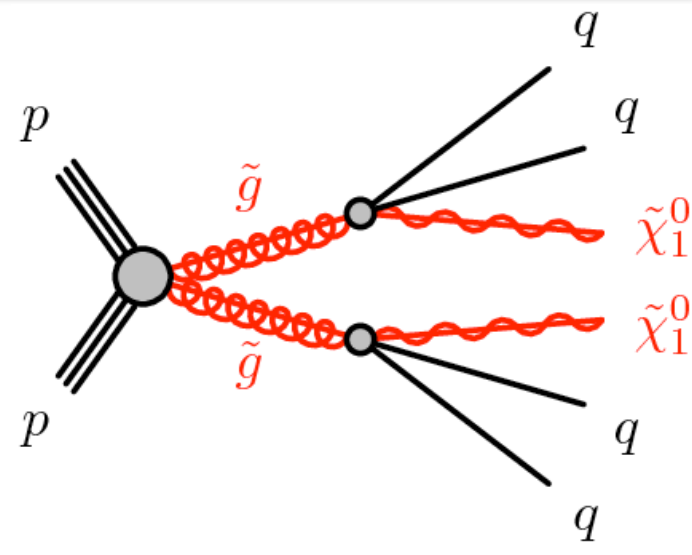

- Z/W+jets, tt and di-boson

$>1.64 \sigma$ exclusion and $5 \sigma$ discovery contours in the $\tilde{g}-\chi_{1}^{0}$ mass plane

$>$ Bands represent $1 \sigma$ uncertainty on the production cross-section

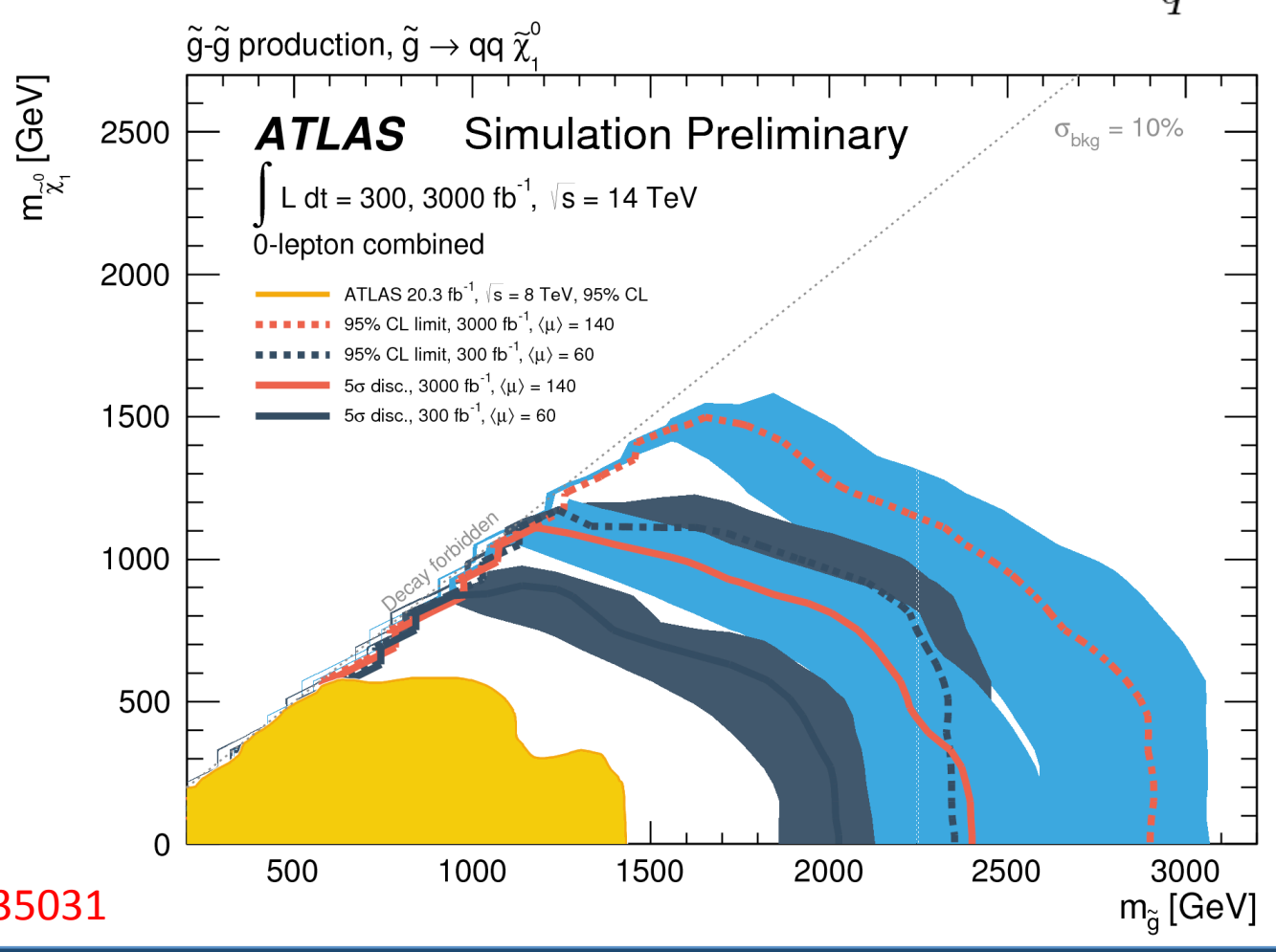




\section{Squark pair production}

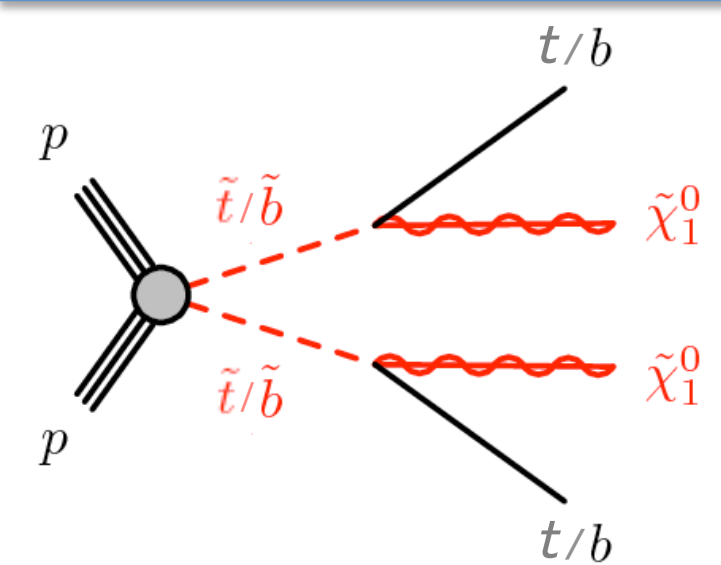

- Require $3^{\text {rd }}$ generation SUSY partners to be light in order to solve the hierarchy problem

- Search for $\tilde{q}$ that exclusively decays $\tilde{q} \rightarrow q \tilde{\chi}_{1}^{0}$

- exclusion/discovery contours are shown for the $\tilde{b}-\chi_{1}^{0}$ and $\tilde{t}-\tilde{\chi}_{1}^{0}$ mass planes:

Sbottom pair production, $\tilde{b}_{1} \rightarrow b \tilde{\chi}_{1}^{0}$, is $=14 \mathrm{TeV}$
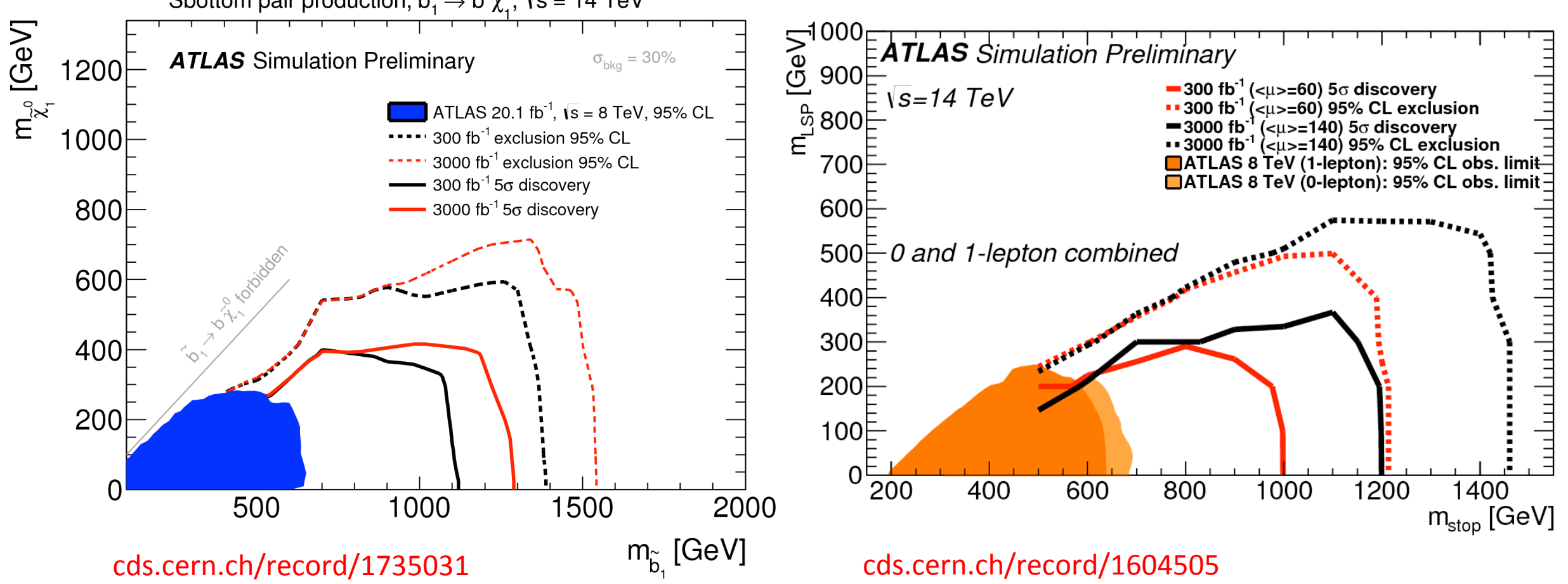

cds.cern.ch/record/1604505 


\section{$\bar{\chi}_{1}^{ \pm} \bar{\chi}_{2}^{0}$ production}

- $\bar{\chi}_{1}^{ \pm}, \bar{\chi}_{2}^{0}$ and $\bar{\chi}_{1}^{0}$ are expected to have masses in the few $100 \mathrm{GeV}$ range

- Multivariate Analysis Techniques (MVA) increase sensitivity, using non-orthogonal cuts in a Boosted Decision Tree

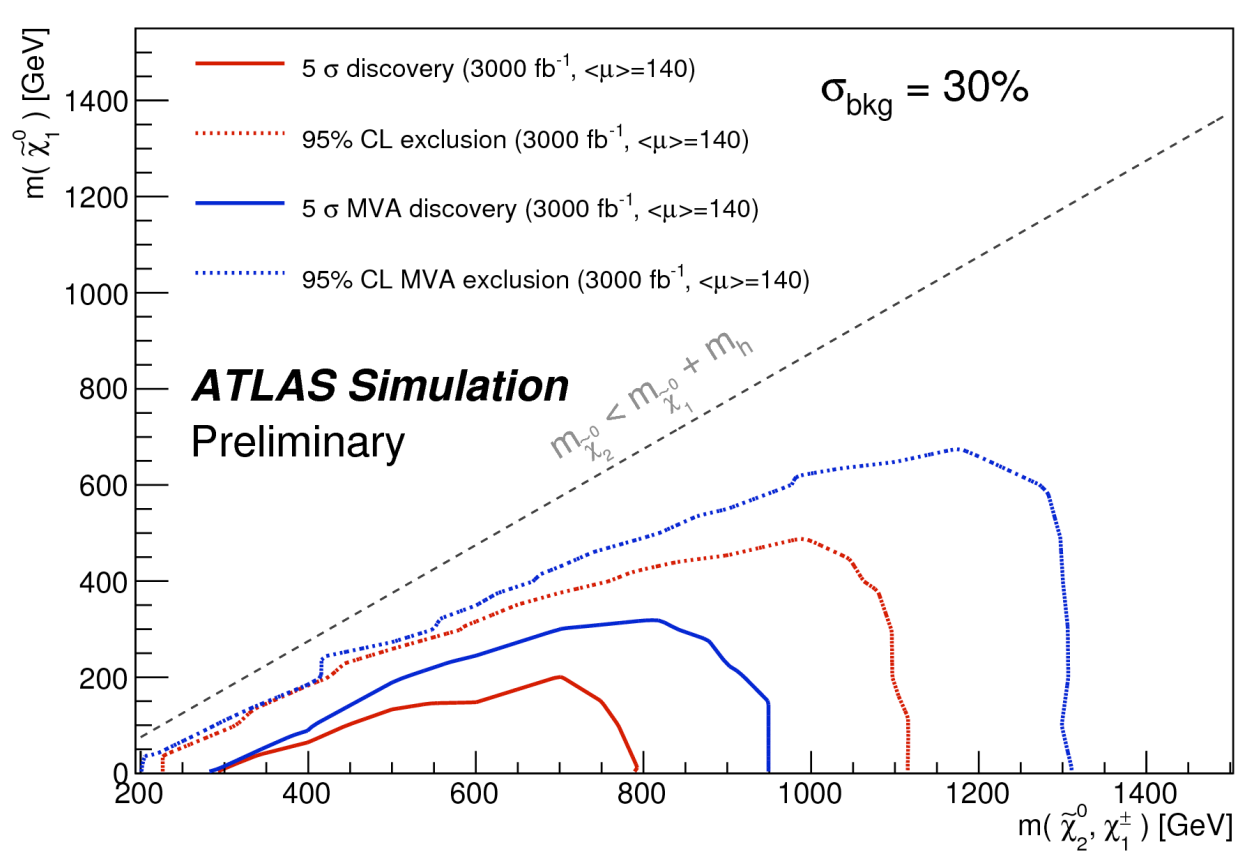

$\left(m_{\bar{x}_{1}}{ }^{ \pm}=m_{\bar{x}_{2}}{ }^{0}\right.$ vs $\left.m_{\bar{x}_{1}}{ }^{0}\right)$ exclusion/discovery limits for count and count and MVA analysis

\section{$\mathrm{W}, \mathrm{h}$ mediated $2 \mathrm{~b}$, 1lep channel}

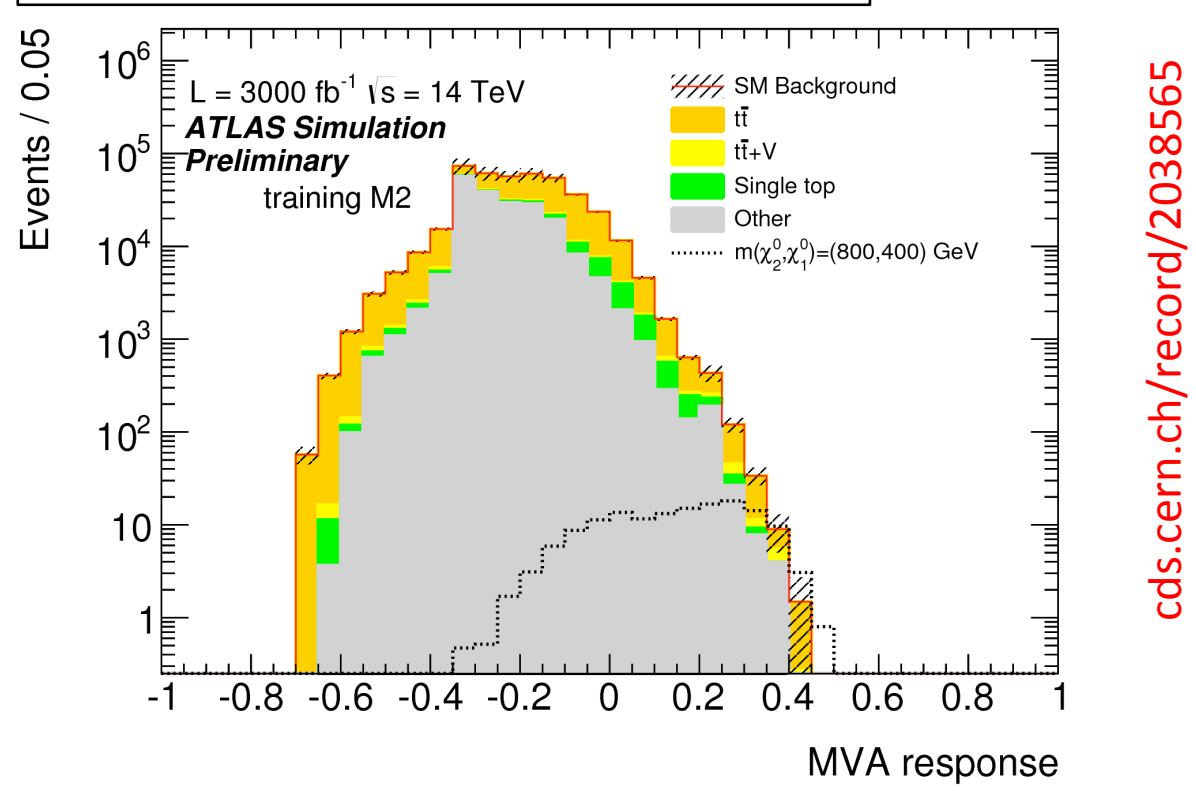

BDT output for training on main backgrounds and given signal masses 


\section{Exotics: Di-jet resonance searches}

A number of new phenomena are expected to modify the di-jet mass distribution, creating a bump on the smooth QCD continuum

- For example excited quarks q* or quantum black holes QBH which decay into two high momentum hadronic jets

- Assumes $q^{*} \rightarrow q+$ gauge bosons decays

- $Q B H$ decays to all allowed two-body final states, simulated using the BlackMax generator see Phys. Rev. D 77 (2008) 076007

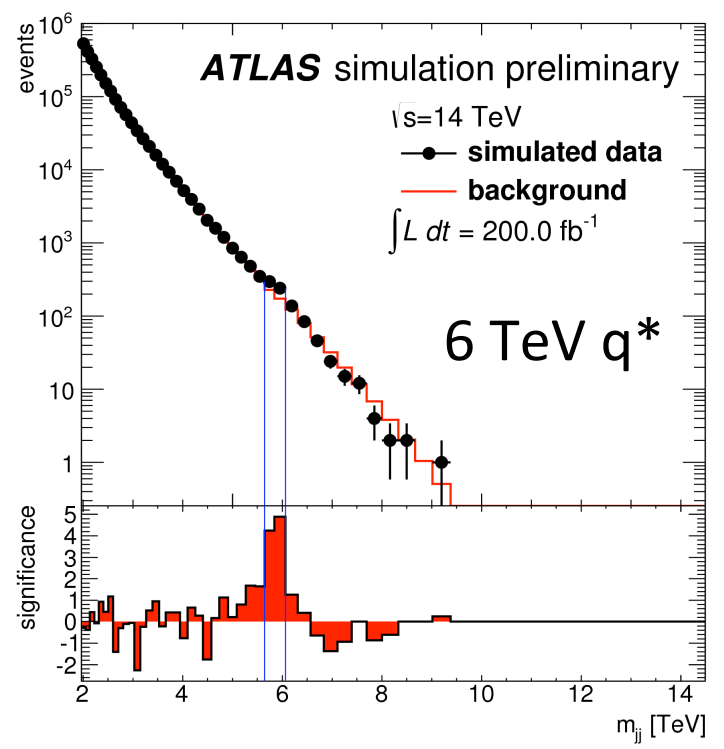

Expected limits Limits are calculated from projected sensitivity and theoretical expectation on $\sigma \times B R$ :

\begin{tabular}{c|c|c}
\hline $\begin{array}{c}\text { integrated } \\
\text { luminosity }\left[\mathrm{fb}^{-1}\right]\end{array}$ & $\mathrm{m}_{\mathrm{q}^{*}}[\mathrm{TeV}]$ & $\mathrm{m}_{\mathrm{QBH}}[\mathrm{TeV}]$ \\
\hline 0.1 & 4.0 & 8.2 \\
\hline 1 & 5.0 & 8.9 \\
\hline 5 & 5.9 & 9.2 \\
\hline 25 & 6.6 & 9.7 \\
\hline 300 & 7.4 & 10.0 \\
\hline 3000 & 8.0 & 10.1 \\
\hline
\end{tabular}

Equivalent to current $20.3 \mathrm{fb}^{-1} 8 \mathrm{TeV}$ limits

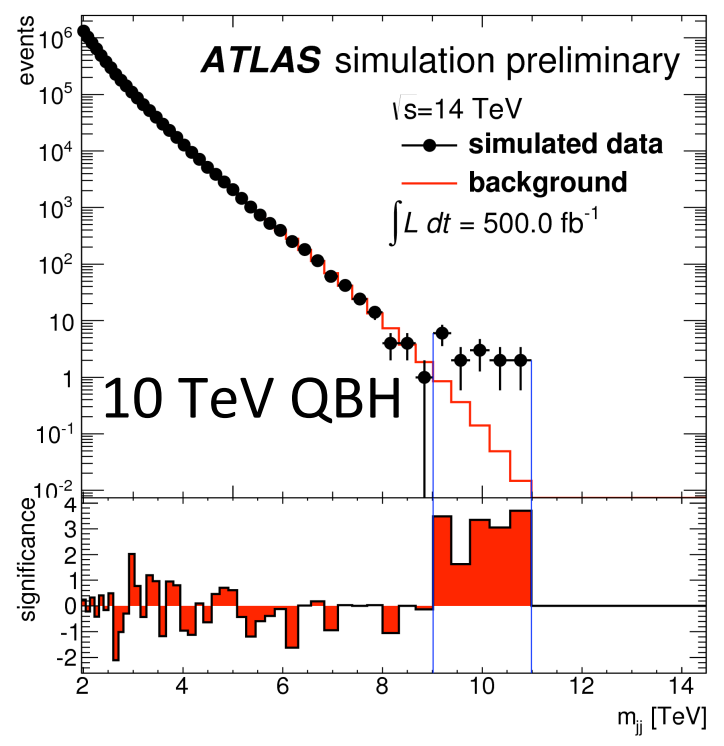




\section{Flavour Changing Neutral Currents}

cds.cern.ch/record/1564937

- FCNC are allowed but highly suppressed in SM loop diagrams, expected to have $B R^{\sim} 10^{-12}$-> inaccessible even at HL-LHC

- Therefore any observation would be a strong sign of new physics

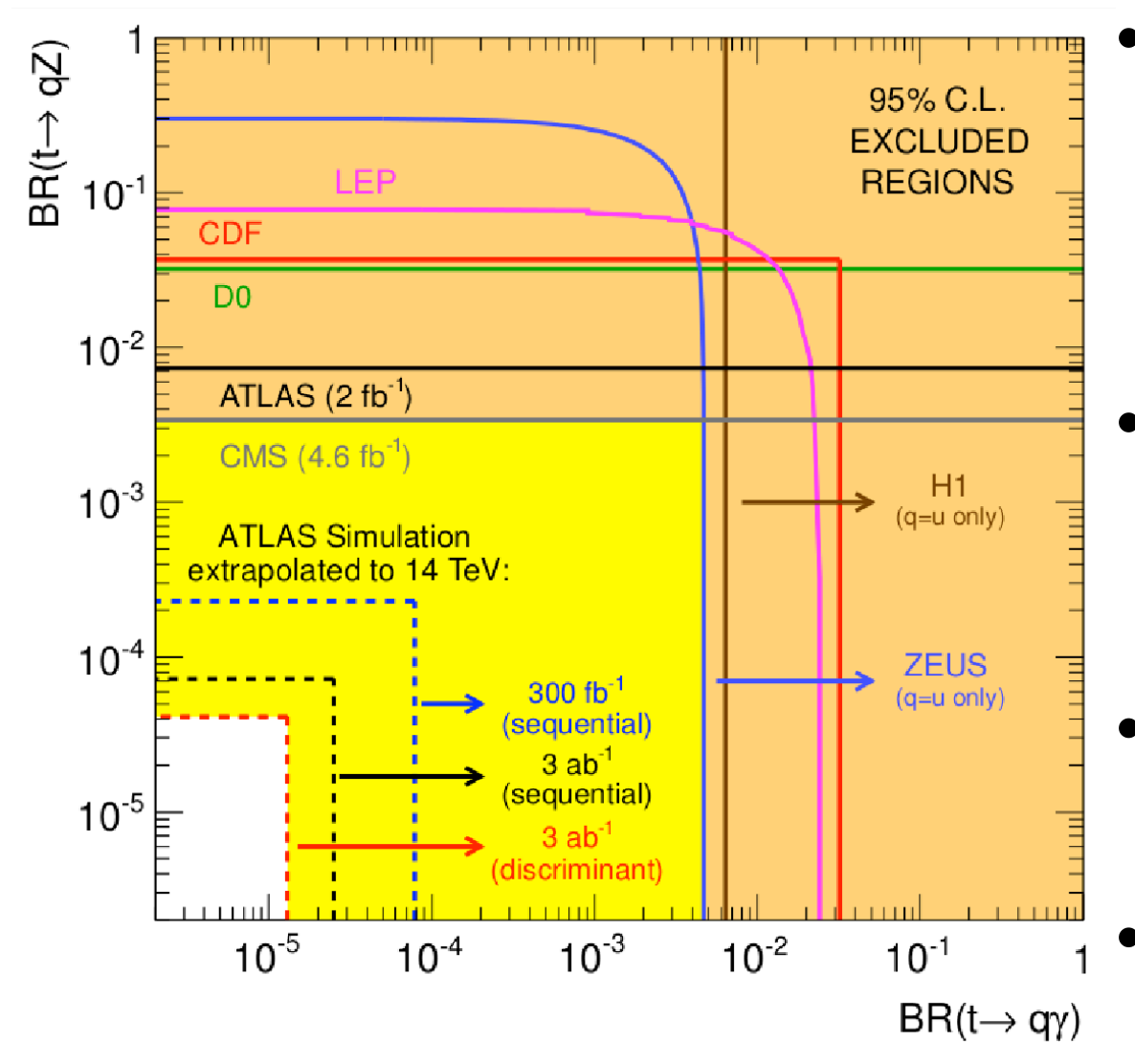

- BSM enhancement of the BR could be seen in top decays

- $t->q p$ and $t->q Z$ are considered, where $q$ is either an $u$ or c quark

- Sensitivity is evaluated from model independent effective Lagrangian

- Sequential: extrapolate results from $7 \mathrm{TeV}$ data

- Discriminant: $14 \mathrm{TeV}$ MC analysis, using a likelihood discriminant 


\section{Conclusion}

- With the discovery of the Higgs boson at LHC-Run 1 we enter a new era of particle physics

- Unprecedented collision energy of $14 \mathrm{TeV}$ in Run 2 will vastly increase the scope of New Physics and precision Higgs studies

- The HL-LHC with 10x more luminosity will offer unique opportunities to explore the Higgs sector and probe for high scale New Physics

- the $3000 \mathrm{fb}^{-1}$ dataset at $14 \mathrm{TeV}$ will allow large gains in precision, discovery potential, and will make important, low cross-section measurements possible

- Upgrades to the ATLAS detector are foreseen to ensure optimal performance in harsh experimental environment

- All prospects studies shown are a conservative projection, likely to see significant improvements in analysis techniques

Stay tuned for exciting results to come! 


\section{Backup slides}




\section{Detailed LHC schedule}

\section{LHC roadmap: according to MTP 2016-2020 V1}

LS2 starting in $2019 \quad \Rightarrow 24$ months +3 months BC

LS3 LHC: starting in $2024 \Rightarrow 30$ months +3 months BC Injectors: in $2025 \quad \Rightarrow 13$ months +3 months BC

Physics

Shutdown

Beam commissioning

Technical stop

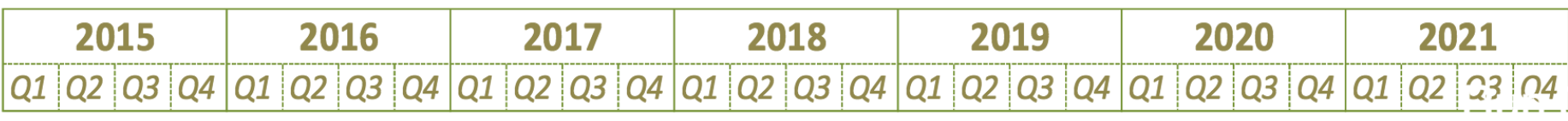

LHC

Injectors

Run 2

LS 2

PHASE 1-

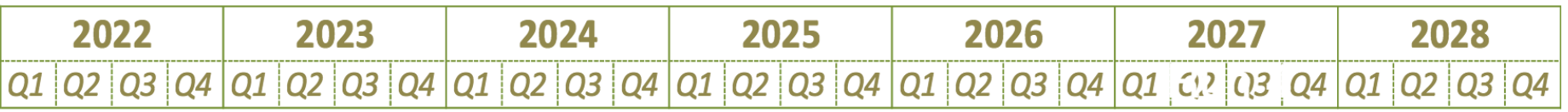

LHC

Injectors

Run 3

LS 3

Run 4

PHASE 2

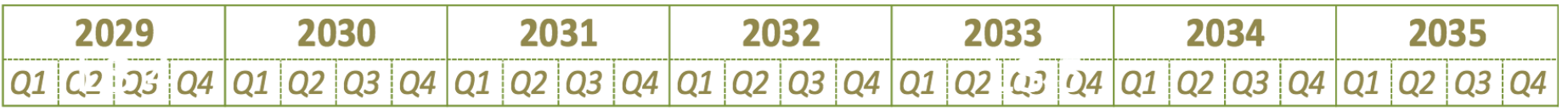

LHC

Injectors

LS 4

Run 5

LS 5

http://lhc-commissioning.web.cern.ch/ (June 2015) 


\section{Detailed LHC schedule}

LHC roadmap: according to MTP 2016-2020 V2

LS2 starting in 2019

LS3 LHC: starting in 2024 Injectors: in 2025
$\Rightarrow 24$ months +3 months $B C$ $\Rightarrow 30$ months +3 months $B C$ $\Rightarrow 13$ months +3 months BC
Physics

Shutdown

Beam commissioning Technical stop

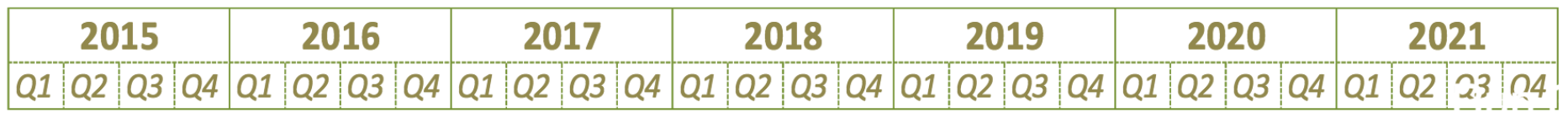

LHC

Injectors

Run 2

LS 2

\section{PHASE 1}

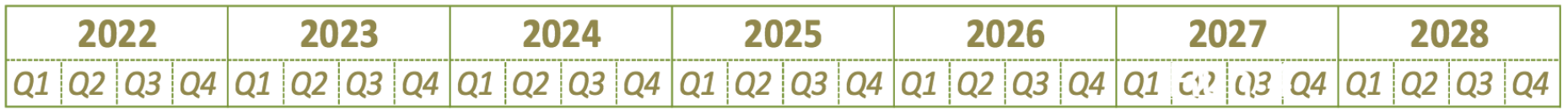

LHC

Injectors

Run 3

LS 3

Run 4

\section{PHASE 2}

\begin{tabular}{|c|c|c|c|c|c|c|c|c|c|c|c|c|c|c|c|c|c|c|c|c|c|}
\hline 2029 & & 20 & & & & 20 & & & & 20 & & & & 20 & & & & & 034 & & 2035 \\
\hline Q1 $C 2: Q 5: Q 4$ & $Q$ & Q2 & Q3 & 4 & 21 & Q2 & 23 & Q4 & Q1 & Q2 & Q3 & Q4 & Q1 & Q2 & (19) & 24 & & 21 Q2 & $2 Q 3$ & Q4 & \begin{tabular}{l|l|l|l} 
Q1 & Q2 & Q3 & Q4
\end{tabular} \\
\hline
\end{tabular}

LHC

Injectors

\section{LS 4}

Run 5

http://lhc-commissioning.web.cern.ch/ (June 2015) 


\section{Bottom-squark pair production}

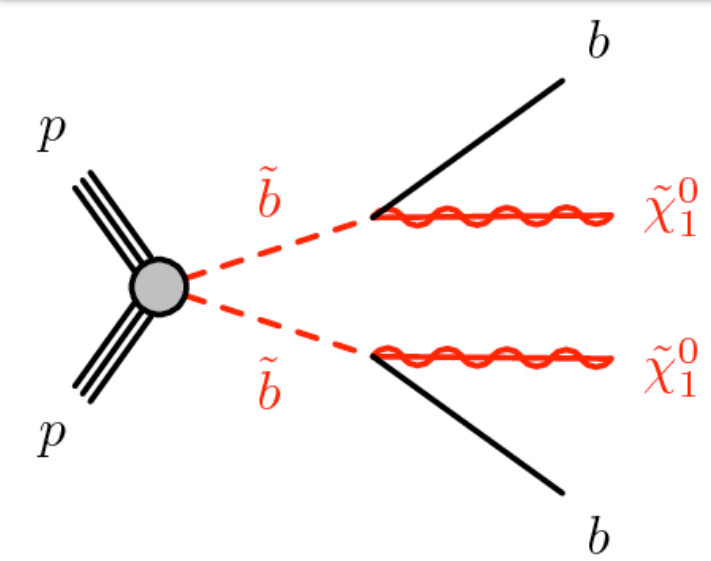

- Require $3^{\text {rd }}$ generation SUSY partners to be light in order to solve the hierarchy problem

- Search for $\tilde{b}$ that exclusively decays $\tilde{b} \rightarrow b \chi_{1}^{0}$

- Event selection based on boost corrected contransverse mass, $\mathrm{m}_{\mathrm{CT}}->$ powerful $t \bar{t}$ veto

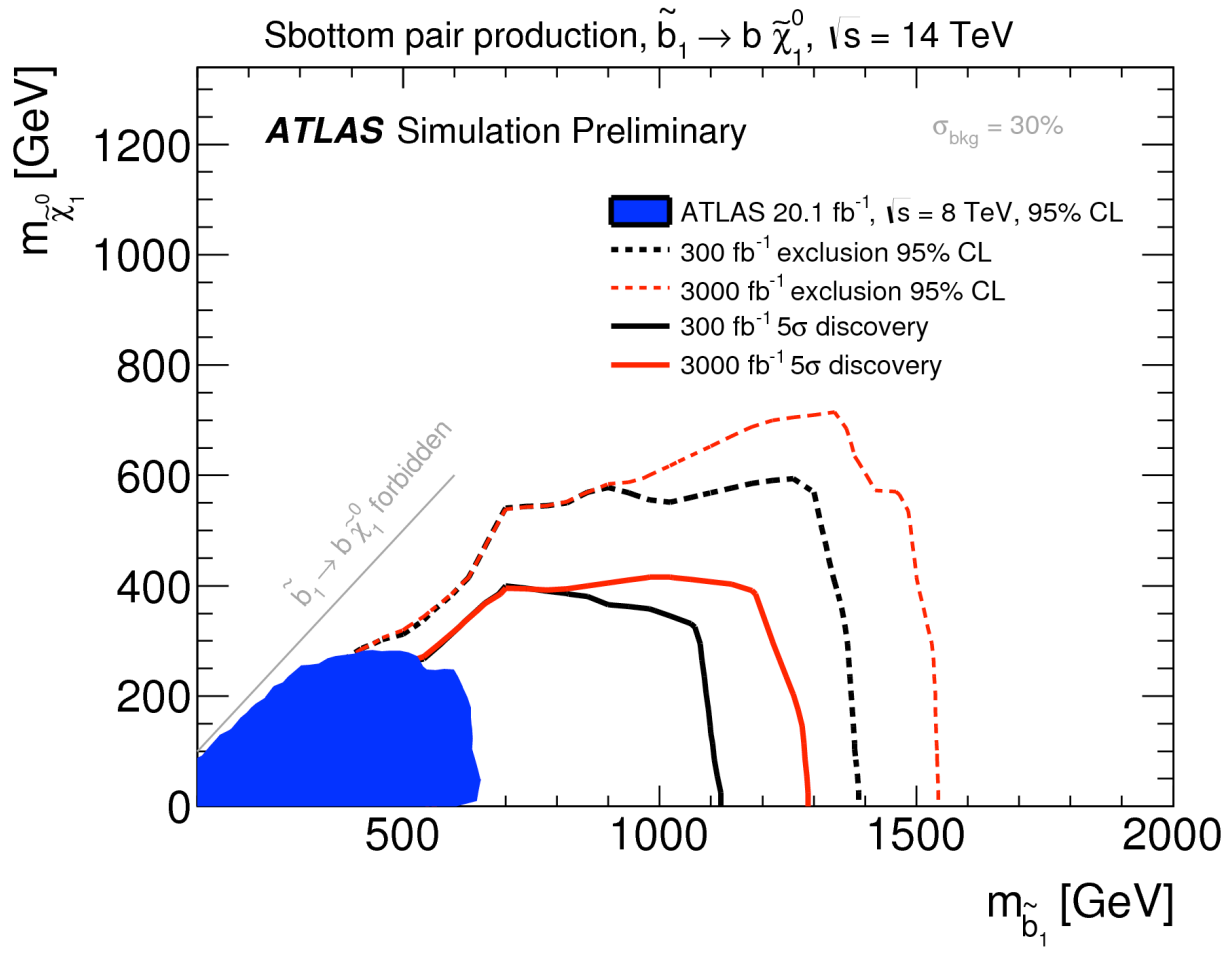

See arXiv:1308.2631 and arXiv:0802.2879

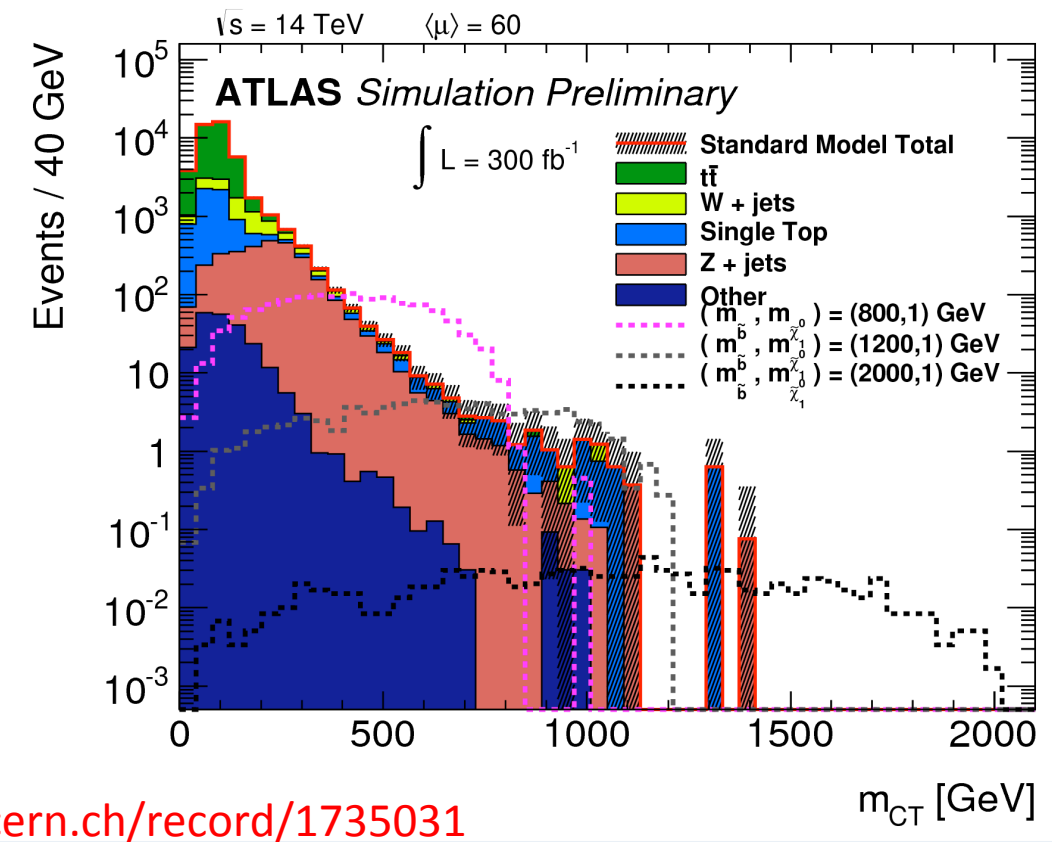




\section{$\bar{\chi}_{1}^{ \pm} \bar{\chi}_{2}^{0}$ production}

\section{- $\bar{\chi}_{1}^{ \pm}$and $\bar{\chi}_{2}^{0}$ are wino-like, $\bar{\chi}_{1}^{0}$ is bino-like}

Missing transverse momentum spectrum for main backgrounds and signal with different mass assumptions

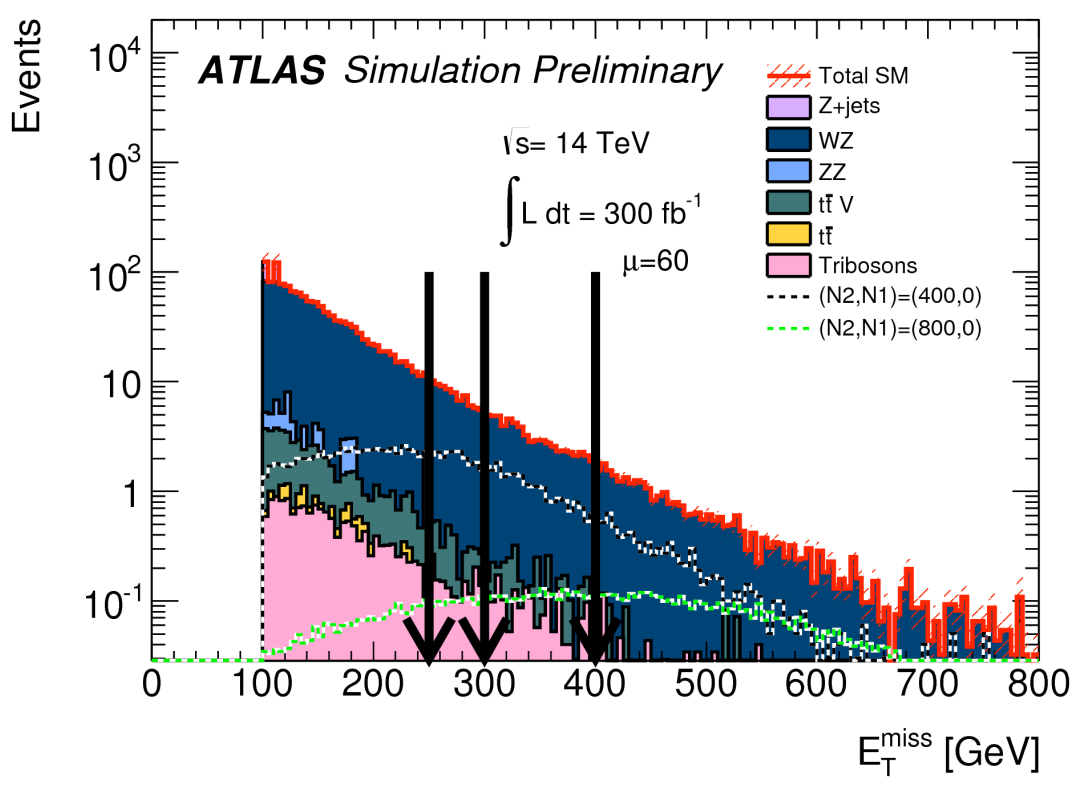

Arrows indicate different signal regions, optimized for exclusion or discovery

$1.64 \sigma$ exclusion \& $5 \sigma$ discovery contours for $\left(m_{\bar{x}_{1}}{ }^{ \pm}=m_{\bar{\chi}_{2}}{ }^{0}\right.$ Vs $\left.m_{\bar{\chi}_{1}}{ }^{0}\right)$
Here, $W, Z$ mediated 3 lepton channel

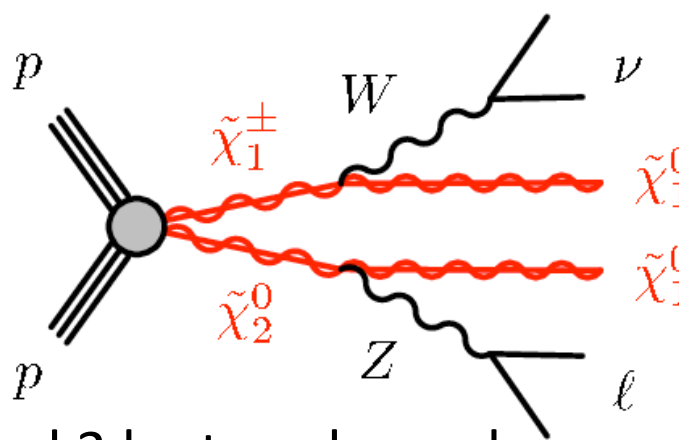




\section{New heavy gauge boson}

- $Z^{\prime} \rightarrow \ell$ resonances search (older study)

- For di-electron or di-muon events, only consider dominant Drell-Yan background

- Assumes a Sequential SM (SSM) $Z_{\text {SSM }}$ with SM fermionic couplings

Limits are calculated from projected sensitivity and theoretical expectation on $\sigma \times B R$
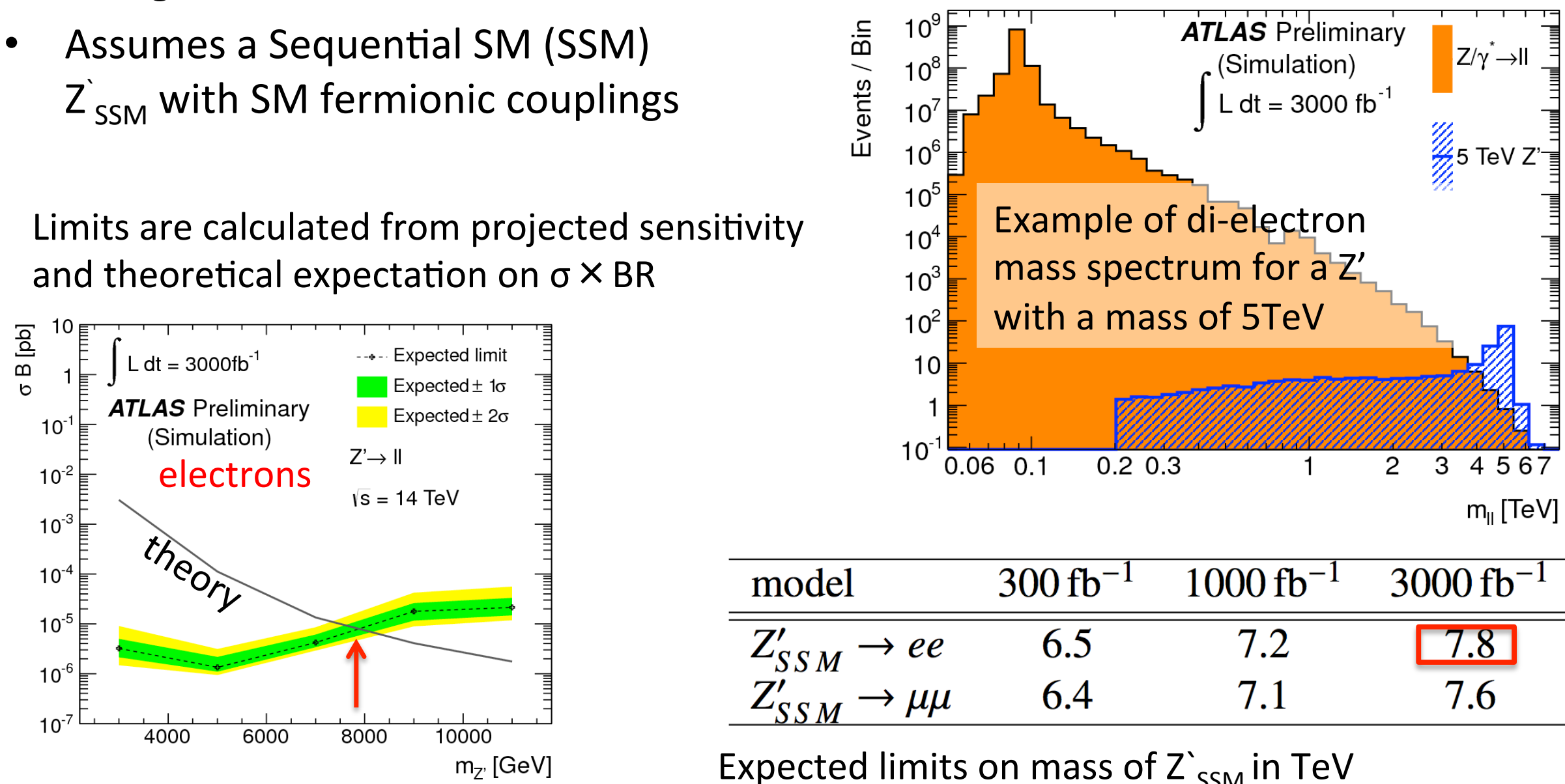

\begin{tabular}{lccc}
\hline model & $300 \mathrm{fb}^{-1}$ & $1000 \mathrm{fb}^{-1}$ & $3000 \mathrm{fb}^{-1}$ \\
\hline \hline$Z_{S S M}^{\prime} \rightarrow e e$ & 6.5 & 7.2 & 7.8 \\
$Z_{S S M}^{\prime} \rightarrow \mu \mu$ & 6.4 & 7.1 & 7.6 \\
\hline
\end{tabular}

Expected limits on mass of $Z_{S S M}$ in $\mathrm{TeV}$ 